\title{
Electrical cross-talk in two-port resonators - the resonant silicon beam force sensor
}

\author{
C. J. van Mullem, H. A. C. Tilmans, A. J. Mouthaan and J. H. J. Fluitman \\ MESA Research Institute, University of Twente, P.O. Box 217, 7500 AE Enschede (Netherlands)
}

\begin{abstract}
An important design consideration in the development of two-port resonant sensors is the electrical cross-talk between the input port and the output port. The overall transfer function $\overline{\boldsymbol{H}}(\mathrm{j} \omega)$ of the two-port sensor is equal to the vectorial sum of a transfer function representing the mechanical behavior and a transfer function representing the electrical cross-talk. The resonant silicon beam force sensor with a piezoelectric driver and a piezoelectric detector is analyzed. Two solutions to reduce the level of cross-talk are presented. Proper adjustment of the value of relevant components in the sensor geometry and/or electrical interruption of the bottom electrode result in a cross-talk level close to the noise level for the frequency range of interest.
\end{abstract}

\section{Introduction}

Resonant silicon sensors, in which the measurand is available as the resonance frequency of a micromechanical structure, offer advantages such as high sensitivity, high accuracy and high stability. Furthermore, the output is a frequency, which is less prone to noise and interference and, owing to its semi-digital nature, it is easily combined with digital circuitry [1]. Also, the realization process of the sensor structure is based on IC technology, which makes it possible to add on-chip circuitry, for instance, to realize an electronic oscillator with the sensor as the frequency-determining building block.

To excite and detect the vibrational motion of the micromechanical structure, two approaches can be used. One uses a single element which combines the excitation and detection of the structure and is designated as a one-port resonator [2]. The other uses separate elements for excitation and detection of the sensor structure, resulting in a two-port resonator [3]. For the excitation and detection, several transduction mechanisms are available [4].

The performance of (resonant) sensors is degraded by several unwanted effects. In the case of a one-port resonator these are due to the parasitic parallel loads, which can obscure the mechanical resonance [2]. For a two-port resonator, a similar effect is caused by the electrical cross-talk between the driving port and the detection port [5]. The electrical cross-talk is a result of capacitive and/or resistive coupling. In the worst situation, the crosstalk will completely obscure the mechanical resonance of the sensor, making detection of the resonant peak impossible. Also, cross-talk may introduce 'anti-resonance' peaks and/or may induce a shift of the frequency at which the amplitude in the transfer function has a maximum. A proper understanding of the causes of electrical cross-talk is required in order to suppress its degrading influence.

In this paper the electrical cross-talk of twoport resonators is investigated further. A piezoelectrically driven resonant beam force sensor [3] is used as an example structure. First, a general model for two-port resonant sensors, including the electrical cross-talk, is discussed using polar diagrams of the different transfer functions. Next, an electrical network, which models the cross-talk of the resonant force sensor, is derived. It is used in conjunction with the mechanical model to simulate the overall sensor transfer function and also to make a comparison with the measured results. Further, the reduction of the cross-talk as a result of changes in the sensor geometry and the sensor configuration is investigated. Measurement results are presented together with simulations. 
Two-port resonator - the transfer function

The overall transfer function $\bar{H}(\mathrm{j} \omega)$ of a twoport resonant sensor can be written as the sum of two contributions: $\bar{M}(\mathrm{j} \omega)$, representing the mechanical behavior, and $\bar{E}(\mathrm{j} \omega)$, representing the electrical cross-talk. This is shown schematically in Fig. 1. In phasor notation the transfer function can be expressed as

$\overline{\boldsymbol{H}}(\mathrm{j} \omega)=\overline{\boldsymbol{M}}(\mathrm{j} \omega)+\bar{E}(\mathrm{j} \omega)$

as illustrated in Fig. 2. In this Figure, the crosstalk $E(\mathrm{j} \omega)$ is assumed to be constant in a narrow frequency range around the fundamental mode. The mechanical transfer function $\bar{M}(\mathrm{j} \omega)$ is approximated by a circle, being characteristic for resonance. The amplitude $|\bar{M}(j \omega)|$ is maximal at a phase angle $\varphi_{M}$ of $90^{\circ}$ occurring at a frequency $\omega=\omega_{1}$. The diameter of the circle depends on the electromechanical transduction factor $\alpha$, as defined by Van Vlerken et al. [6], and on the quality factor of the resonator. High quality factors imply large diameter circles.

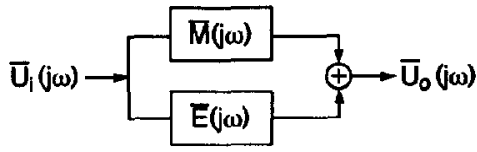

Fig. 1. Block diagram of the overall transfer function of a two-port resonant sensor: $\boldsymbol{A}(\mathrm{j} \omega) \equiv D_{\mathrm{o}}(\mathrm{j} \omega) / D_{\mathrm{i}}(\mathrm{j} \omega)=\overline{\boldsymbol{M}}(\mathrm{j} \omega)+\boldsymbol{E}(\mathrm{j} \omega)$, where $\bar{M}(\mathrm{j} \omega)$ represents the mechanical behavior and $\bar{E}(\mathrm{j} \omega)$ represents the electrical cross-talk. Noise is ignored in this diagram.

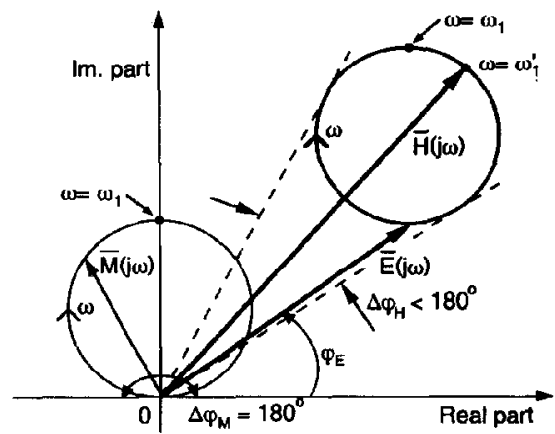

Fig. 2. Theoretical polar diagram of the different transfer functions of a two-port resonator (also see Fig. 1). $E(\mathrm{j} \omega)$ is assumed to be constant in the frequency range around the fundamental mode. Noise is ignored in this diagram.
From eqn. (1) expressions for the magnitude $|\overline{\boldsymbol{H}}(\mathrm{j} \omega)|$ and phase $\varphi_{H}$ can be derived:

$$
\begin{aligned}
|\overline{\boldsymbol{H}}(\mathrm{j} \omega)|= & |\overline{\boldsymbol{M}}(\mathrm{j} \omega)|\left[\left(\cos \varphi_{M}+\frac{|\bar{E}(\mathrm{j} \omega)|}{|\overline{\boldsymbol{M}}(\mathrm{j} \omega)|} \cos \varphi_{E}\right)^{2}\right. \\
& \left.+\left(\sin \varphi_{M}+\frac{|\boldsymbol{E}(\mathrm{j} \omega)|}{|\overline{\boldsymbol{M}}(\mathrm{j} \omega)|} \sin \varphi_{E}\right)^{2}\right]^{1 / 2}
\end{aligned}
$$

$\varphi_{H}=\arg \overline{\boldsymbol{H}}(\mathrm{j} \omega)$

$$
=\arctan \left(\frac{\sin \varphi_{M}+\frac{|\boldsymbol{E}(\mathrm{j} \omega)|}{|\overline{\boldsymbol{M}}(\mathrm{j} \omega)|} \sin \varphi_{E}}{\cos \varphi_{M}+\frac{|\overline{\boldsymbol{E}}(\mathrm{j} \omega)|}{|\overline{\boldsymbol{M}}(\mathrm{j} \omega)|} \cos \varphi_{E}}\right)
$$

where $\varphi_{M}$ and $\varphi_{E}$ are the phase angles of the mechanical and electrical transfer function, respectively. In Fig. 3 a typical Bode plot of $\bar{H}(\mathrm{j} \omega)$ is given. In this Figure, as opposed to Fig. 2, $\bar{E}(\mathrm{j} \omega)$ is now frequency dependent. From eqns. (2) and (3) it can be seen that for small values of the ratio $|\overline{\boldsymbol{E}}(\mathrm{j} \omega)| /|\overline{\boldsymbol{M}}(\mathrm{j} \omega)|$ the electrical cross-talk can be

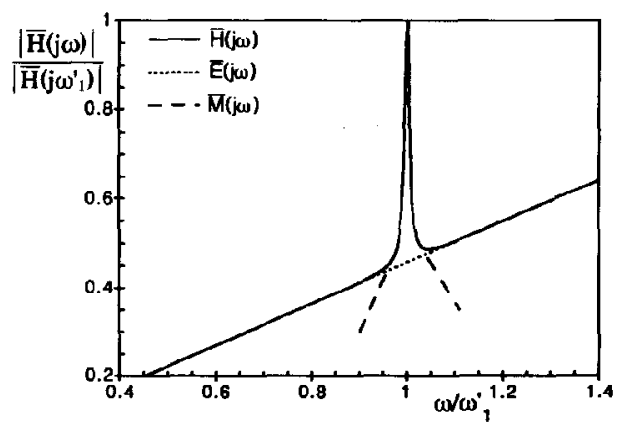

(a)

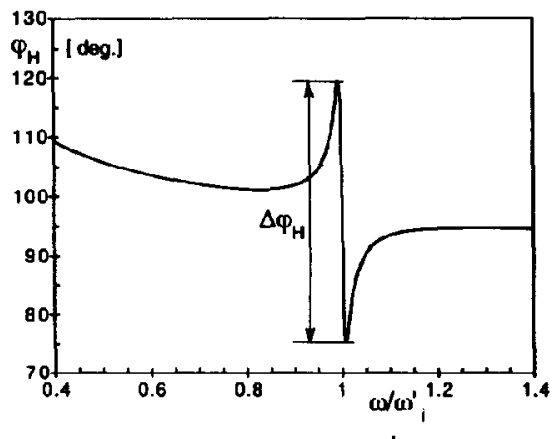

(b)

Fig. 3. Simulated typical Bode plots: (a) normalized modulus; (b) phase angle. $\Delta \varphi$ is the maximum observed phase shift as indicated in Fig. 2. 
ignored and $|\bar{H}(\mathrm{j} \omega)|$ and $\varphi_{H}$ will approach $|\overline{\boldsymbol{M}}(\mathrm{j} \omega)|$ and $\varphi_{M}$, respectively.

The electrical cross-talk introduces an off-set of the resonance loop in the complex plane. This has several consequences. First of all, the frequencies at which the amplitudes of $\bar{M}(\mathrm{j} \omega)$ and $\bar{H}(\mathrm{j} \omega)$ are maximal are no longer the same. For the example shown in Fig. 2, the maximum of $|\overline{\boldsymbol{H}}(\mathrm{j} \omega)|$ occurs at a (slightly) higher frequency, $\omega_{1}^{\prime}$, than the frequency $\omega_{1}\left(<\omega_{1}^{\prime}\right)$ at which $\overline{\boldsymbol{M}}(\mathrm{j} \omega)$ has its maximum. The relative change in magnitude also becomes smaller due to the cross-talk. Furthermore, the expected phase shift $\Delta \varphi$ of $180^{\circ}$ in the frequency interval around resonance will be smaller (see Fig. 3). All this makes the mechanical resonance less pronounced, calling for compensation and/or reduction of the cross-talk.

Two approaches can be used to minimize the electrical cross-talk. First, a compensation technique can be chosen. An example is an on-chip differential electrical design where another identical electrical structure is realized in addition to the sensor structure. Here, the total output signal is given by the sensor output minus the output of the electrical structure in order to eliminate the electrical cross-talk. Secondly, the sensor structure and/or the sensor geometry can be optimized. This solution will be described further in the next section.

\section{The resonant silicon beam force sensor [3]}

\section{The structure}

A schematic drawing of the sensor is shown in Fig. 4. The heart of the sensor is a silicon beam. Piezoelectric layers of $\mathrm{ZnO}$ are used for the excitation and detection of the vibration. A double

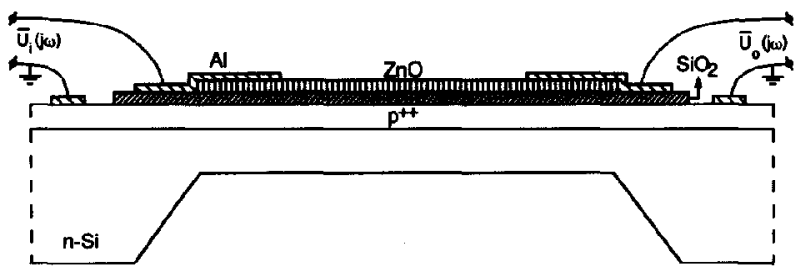

Fig. 4. Cross-sectional drawing of the resonant silicon beam force sensor [3]. Beam dimensions: length, $6 \mathrm{~mm}$; width, $0.4 \mathrm{~mm}$; thickness, $20 \mu \mathrm{m}$. layer of $\mathrm{ZnO}$ and $\mathrm{SiO}_{2}$ is sandwiched between a $\mathrm{p}^{++}$-bottom and an aluminum top electrode. The $\mathrm{SiO}_{2}$ is used to insulate the top from the bottom electrode and forms, in combination with the $\mathrm{ZnO}$ layer, a dielectric medium for the piezoelectric excitation and detection $[7,8]$.

\section{Theoretical model of the electrical and mechanical transfer function}

The model for the electrical transfer function of the resonant force sensor is based on the crosssection of Fig. 4. An equivalent electrical lumpedelement network of the sensor is shown in Fig. 5. The bottom electrode is modelled with five resistors $\left(R_{1-5}\right)$. The $\mathrm{SiO}_{2}$ and the $\mathrm{ZnO}$ layers are modelled as a parallel combination of a capacitor $\left(C_{\mathrm{SiO}_{2}}\right.$ and $\left.C_{\mathrm{ZnO}}\right)$ and a resistor $\left(R_{\mathrm{SiO}_{2}}\right.$ and $\left.R_{\mathrm{ZnO}}\right)$. The model assumes that the $\mathrm{ZnO}$ layer is completely depleted [8]. The input capacitor $\left(C_{\mathrm{i}}\right)$ represents the capacitance between the top and the bottom electrode and $R_{\mathrm{i}}$ the d.c. resistance of the $\mathrm{SiO}_{2}$ layer sandwiched between the aluminum and the $\mathrm{p}^{++}$-layer. In the model the parasitic impedances caused by wires, probes and housing are not taken into account.

The origin of the electrical cross-talk can be explained with the circuit of Fig. 5. An input voltage will produce an output voltage because of the electrical connection between the input port and the output port. The finite resistivity of the bottom electrode means that node $A$ is not at ground potential. Theoretically, if node $\mathrm{A}$ is grounded the cross-talk will be completely suppressed.

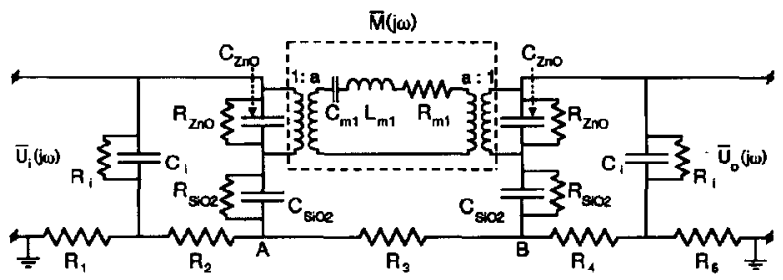

Fig. 5. Equivalent electrical network of the resonant beam force sensor for a floating substrate. $C_{\mathrm{m} 1}$ and $L_{\mathrm{m} 1}$ are functions of the specific mass and the beam dimensions and $R_{\mathrm{ml}}$ represents the energy losses [6]. The model is valid for a narrow frequency range around the fundamental frequency $\omega_{1}$. The transformer couples energy from the electrical domain to the mechanical domain and vice versa. The transformer turns ratio $a$ depends on the electromechanical transduction factor $a$ and on the mode shape [6]. 
The mechanical transfer function $\bar{M}(\mathrm{j} \omega)$ of the resonant force sensor is modelled by a lumpedelement model [6] as shown in Fig. 5 and applies for a narrow frequency range around the fundamental vibrational mode. With ideal transformers the electrical input is converted into the mechanical domain, where each vibrational mode $n$ is represented by a modal mass $L_{\mathrm{m}, n}$, a compliance $C_{\mathrm{m}, n}$ and a resistance $R_{\mathrm{m}, n}$, and back into the electrical domain. The model can easily be extended to include other modes. The piezoelectric transduction is reflected in the transformer turns ratio $a$. The efficiency of the transduction is decreased since only a fraction of the input voltage will appear across the $\mathrm{ZnO}$ layer. This fraction can be increased if the $\mathrm{ZnO}$ layer is deposited directly on top of the $\mathrm{p}^{++}$-bottom electrode [8]; this way the voltage drop across the oxide layer is avoided.

\section{Measured and simulated overall transfer function}

In Fig. 6 a measured and simulated polar plot of $\overline{\boldsymbol{H}}(\mathrm{j} \omega)$ is given. Both plots clearly indicate that $\overline{\boldsymbol{H}}(\mathrm{j} \omega)$ is equal to the sum of the two transfer functions $\overline{\boldsymbol{M}}(\mathrm{j} \omega)$ and $\overline{\boldsymbol{E}}(\mathrm{j} \omega)$, (compare Fig. 2). The electromechanical transduction factor $\alpha$ is adjusted in order to obtain the same loop diameter for the simulated plot as for the measured plot. The measured $\Delta \varphi_{H}$ is about $40^{\circ}$ and the maximum $\left|\overline{\boldsymbol{H}}\left(\mathrm{j} \omega_{1}^{\prime}\right)\right|$ is $-75 \mathrm{~dB}$. The unloaded resonance frequency $f_{1}^{\prime}$ is around $4.8 \mathrm{kHz}$, which is close to the theoretical value of $4.9 \mathrm{kHz}$.

\section{Reduction of the electrical cross-talk}

To reduce the influence of $\overline{\boldsymbol{E}}(\mathrm{j} \omega)$ on $\overline{\boldsymbol{H}}(\mathrm{j} \omega)$ the sensor structure itself is investigated. Looking at

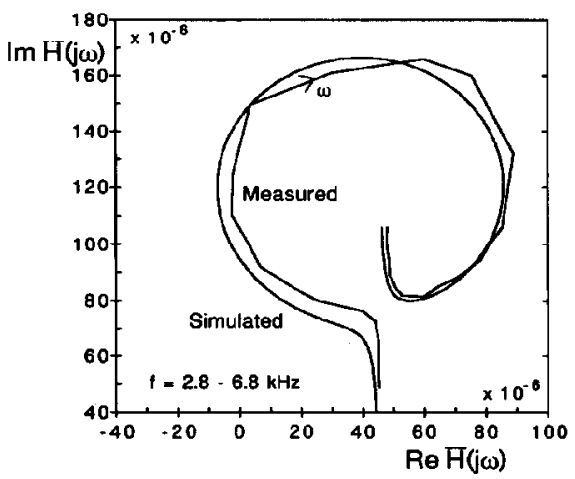

Fig. 6. Measured and simulated polar diagram of $\vec{H}(\mathrm{j} \omega)$ of the sensor shown in Fig. 4. the equivalent network for the electrical cross-talk in Fig. 5, reduction can be achieved by:

- reducing the series resistance from point $A$ and/ or $\mathbf{B}$ to ground;

- reducing the capacitances to decrease the voltage on node $A$ (B);

- increasing the impedance between points $A$ and B;

- low leakage dielectric layers to increase the d.c. parallel resistance.

Implementation of the above is possible by the following means.

(1) Use of other materials. For example, a metal can be used instead of a $\mathrm{p}^{++}$-layer for the bottom electrode in order to lower the resistivity of the bottom electrode. This way, node A will he grounded. A disadvantage, however, is the lack of stress compensation, since the tensile stress in the $\mathrm{p}^{++}$-bottom electrode is used to compensate the compressive stress of the $\mathrm{ZnO}$ layer [3]. Also, materials can be chosen which have a higher d.c. resistance than CVD oxide, for example, thermal oxide or LPCVD silicon nitride.

(2) Change of the sensor geometry. The value of relevant components in the geometry can be changed to reduce the voltage at node $A$. These components are the capacitor $C_{\mathrm{i}}$ and the resistors $R_{1}$ and $R_{5}$. The other components $\left(C_{\mathrm{ZnO}}, C_{\mathrm{SiO}_{2}}\right.$ and $R_{2-4}$ ) are dictated by the required mechanical characteristics, making adjustments less flexible. The modified geometry will have a smaller bonding pad area and, furthermore, the aluminum contact for the bottom electrode is moved closer to the end of the sensor beam.

(3) Change of the sensor configuration. Interruption of the bottom electrode by means of two anti-serial $\mathrm{p}-\mathrm{n}$ junctions will increase $R_{3}$ and thus provides a high-resistance path between points $A$ and B (see Fig. 7). The sensor is realized in an n-type silicon wafer. As indicated before, the tensile stress in the $\mathrm{p}^{++}$-layer compensates the compressive stress in the $\mathrm{ZnO}$ layer. For a small interruption $(20 \mu \mathrm{m})$, stress compensation is still accomplished.

Solutions (2) and (3) have actually been realized and the measured results are shown in Fig. 8. Interruption of the bottom electrode gives a stronger reduction of the cross-talk. The modified geometry and interruption of the bottom electrode result in a cross-talk level of the order of the noise level for the frequency range of interest, that is, 


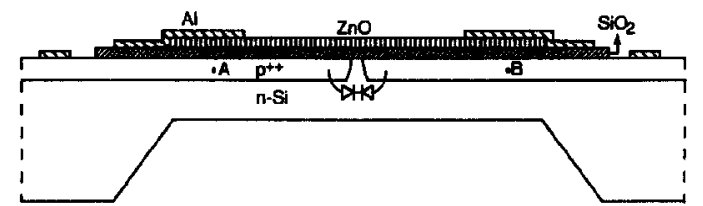

Fig. 7. Cross-sectional drawing of the resonant silicon beam force sensor with the bottom electrode interrupted by means of two antiserial $\mathbf{p}-\mathbf{n}$ junctions.

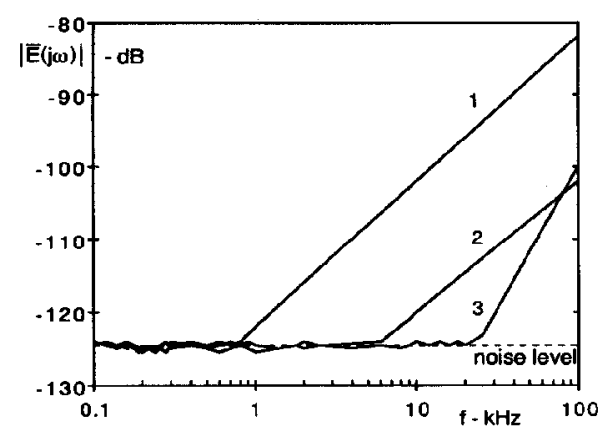

Fig. 8. Measured and simulated electrical transfer function: (1) original design; (2) after adjusting $C_{\mathrm{i}}, R_{1}$ and $R_{5}$; (3) after interrupting the bottom electrode.

$1-30 \mathrm{kHz}$. The second solution has also been simulated including the mechanical model. The results are shown in Fig. 9. It is clearly seen that the level of cross-talk is reduced, which will move the resonance loop closer to the origin. The crosstalk still affects the phase shift but the overall phase shift approaches $180^{\circ}$. An additional improvement is the increase of the diameter of the circle. The change of geometry increases the frac-

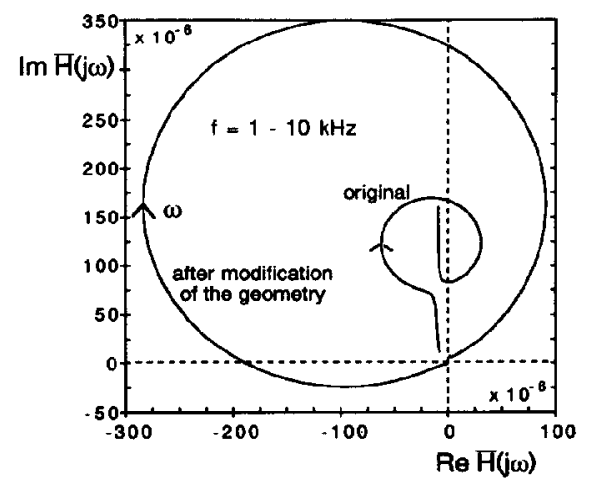

Fig. 9. Simulated overall sensor transfer function for two different levels of cross-talk. tion of the input voltage which appears across the $\mathrm{ZnO}$ capacitor. Both the decrease of the crosstalk and the larger diameter imply an increase in the relative change of magnitude.

\section{Conclusions}

Besides the mechanical coupling, $\bar{M}(\mathrm{j} \omega)$, between the input and output port of two-port resonant sensors, an electrical coupling, $\bar{E}(\mathrm{j} \omega)$, due to cross-talk, exists. The cross-talk is caused by capacitive and/or resistive coupling between the driving port and the detection of the sensor. The overall transfer function $\overline{\boldsymbol{H}}(\mathrm{j} \omega)$ can be written as the vectorial sum of $\overline{\boldsymbol{M}}(\mathrm{j} \omega)$ and $\overline{\boldsymbol{E}}(\mathrm{j} \omega)$. In a polar diagram, $\bar{H}(\mathrm{j} \omega)$ is shifted away from the origin due to the electrical component. In a Bode plot, $\bar{E}(\mathrm{j} \omega)$ manifests itself as a decrease of the maximum phase shift of $\boldsymbol{H}(\mathrm{j} \omega)$ around resonance and, further, by a decrease of the relative change in magnitude. Both effects will make detection of the mechanical resonance more difficult.

The resonant silicon-beam force sensor with piezoelectric excitation and detection of the vibrational motion of the beam has been analyzed. In the original design, the electrical cross-talk limitated the maximum phase shift to about $40^{\circ}$. To reduce the cross-talk level and increase the phase shift of $\overline{\boldsymbol{H}}(\mathrm{j} \omega)$, two solutions were investigated. First, the value of relevant components in the sensor geometry were changed. Second, the bottom electrode was interrupted by means of two anti-serial, $\mathrm{p}-\mathrm{n}$ junctions. Applying both solutions at the same time results in a cross-talk level close to the noise level for the frequency range of interest. Also, the overall phase shift approaches $180^{\circ}$ due to reduction of the electrical cross-talk.

\section{Acknowledgements}

The authors wish to thank Dr F. R. Blom and P. J. C. Zweers for their contribution to this work. These investigations in the program of the Fundamental Research on Matter (FOM) were supported by the Dutch Technology Foundation (STW). 


\section{References}

1 S. Middelhoek, P. J. French, J. H. Huijsing and W. J. Lian, Sensors with digital or frequency output, Sensors and Actuators, 15 (1988) 119-133.

2 H. A. C. Tilmans, D. J. Yntema and J. H. J. Fluitman, Single element excitation and detection of (micro-)mechanical resonators. Proc. 6th Int. Conf. Solid-State Sensors and Actuators (Transducers 91), San Francisco, CA, USA, June 24-27, 1991, pp. 533-537.

3 C. J. van Mullem, F. R. Blom, J. H. J. Fluitman and M. Elwenspoek, Piezoelectrically driven silicon beam force sensor, Sensors and Actuators, A25-A27 (1991) 379-383.

4 M. Elwenspoek, F. R. Blom, S. Bouwstra, T. S. J. Lammerink, F. C. M. van de Pol, H. A. C. Tilmans, Th. J. A. Popma and J. H. J. Fluitman, Transduction mechanisms and their applications in micromechanical devices, IEEE Proc. Conf. Micro Electro Mechanical Systems (MEMS '89), February 1989, pp. 126-132.

5 R. T. Howe and R. S. Muller, Resonant-microbridge vapor sensor, IEEE Trans. Electron Devices, ED-33 (1986) 499-506.

6 J. J. L. M. van Vlerken, S. Bouwstra, F. R. Blom, J. H. J. Fluitman and P. C. Breedveld, Finite-mode bond-graph model of a resonant silicon-beam force sensor, Int. J. Modelling Simulation, 12 (2) in press.

7 F. R. Blom, D. J. Yntema, F. C. M. van de Pol, M. Elwenspoek, J. H. J. Fluitman and Th. J. A. Popma, Thin-film $\mathrm{ZnO}$ as micromechanical actuator at low frequencies, Sensors and Actuators, 121 A23 (1990) $226-228$.

8 F. R. Blom, F. C. M. van de Pol, G. Bauhuis and Th. J. A. Popma, (R. f. planar magnetron sputtered) $\mathrm{ZnO}$ films II: electric properties, Thin Solid Films, 204 (1991) 365-376. 